\title{
Paralytic ileus due to a novel anticancer drug, nab-paclitaxel: A case report
}

\author{
XIAO-DONG JIAO ${ }^{1 *}$, XIU LUO $^{2 *}$, WEN-XING QIN ${ }^{1 *}$, LING-YAN YUAN $^{1}$ and YUAN-SHENG ZANG ${ }^{1}$ \\ ${ }^{1}$ Department of Medical Oncology, Changzheng Hospital, Shanghai 200070; \\ ${ }^{2}$ Department of Hematology, Tongji Hospital of Tongji University, Shanghai 200065, P.R. China
}

Received October 12, 2015; Accepted January 28, 2016

DOI: $10.3892 / \mathrm{mco} .2016 .782$

\begin{abstract}
Nab-paclitaxel is a recently emerged chemotherapy drug, which is widely used for the treatment of multiple types of cancer. The prospects of this novel drug are very bright as a result of its higher efficacy and lower toxicity compared with paclitaxel. Hence, the side effect, even if rare, require attention in clinical practice. The present study described an unusual case of nab-paclitaxel-associated paralytic ileus. To the best of our knowledge, this is the first report to demonstrate that nab-paclitaxel may lead to acute intestinal obstruction. Since nab-paclitaxel will be used more frequently, this unusual side effect might be encountered by a clinical oncologist and must be treated correctly. This is the first reported case, to the best of our knowledge, of paralytic ileus caused by nab-paclitaxel, which will be widely used as a novel anticancer drug.
\end{abstract}

\section{Introduction}

Nab-paclitaxel is a newly developed chemotherapy drug, which is albumin-bound paclitaxel nanoparticles (1). Compared with ordinary paclitaxel, nab-paclitaxel is highly soluble and can easily reach potential target tumor tissue through the bloodstream, exerting its broad-spectrum antitumor activity (2). It is now commonly used in breast cancer, pancreatic cancer, lung cancer and gastric cancer (3-6). In clinical practice, the infusion time for nab-paclitaxel is shorter and the incidence of allergic reactions is notably lower compared with ordinary paclitaxel, which significantly potentiates its clinical efficacy (7). However, the safety of this agent remains to be fully understood, and the side effects have rarely been reported. Although there several previous studies focussing on the side effect of this novel drug, and

Correspondence to: Dr Yuan-Sheng Zang, Department of Medical Oncology, Changzheng Hospital, 64 Hetian Road, Shanghai 200070, P.R. China

E-mail: doctorzangys@163.com

${ }^{*}$ Contributed equally

Key words: nab-paclitaxel, paralytic ileus, complication the majority of the mentioned side effects were edema, heart failure, asthenia, neutropenia and neuropathy (8-12), another previous study reported a rare case of capillary leak syndrome and pulmonary hypertension following treatment with nab-paclitaxel (13). However, in consideration of the limited clinical administration and short practice time, certain rare side effects must exist, which may not have been revealed in clinical trails. The present study reported an unusual case of nab-paclitaxel-associated paralytic ileus. Although this case accepted gemcitabine and nab-paclitaxel at the same time, considering well-demonstrated side effects of gemcitabine (14), the present study deduced that the rare paralytic ileus was associated with nab-paclitaxel. This case was accurately diagnosed and recovered well following the effective treatments. The present case provided additional evidence for the probable adverse effect of this novel drug, which may further guide our clinical practice.

\section{Case report}

A 65-year-old male with pancreatic cancer was admitted to the Department of Medical Oncology, Changzheng Hospital (Shanghai, China). He was diagnosed with pancreatic adenocarcinoma and had undergone a radical surgery 17 months previously (Fig. 1). However, the patient suffered from tumor recurrence and metastasis, according to a recent computed tomography (CT) examination, which revealed tumor recurrence, and metastasis to the spleen and retroperitoneal lymph node. According to the latest research (4), the present case study selected nab-paclitaxel plus gemcitabine as the first-line chemotherapy (nab-paclitaxel, $130 \mathrm{mg} / \mathrm{m}^{2}$ intravenous infusion days 1 and 8 and gemcitabine $1,000 \mathrm{mg} / \mathrm{m}^{2}$ intravenous infusion days 1 and 8 , every 3 weeks).

After 3 days of the second cycle of chemotherapy (November $24^{\text {th }} 2014$ ), the patient complained of sudden and sustained abdominal pain with bloating and reduced anal exhaust. Physical examinations revealed abdominal distension, cullen and lower abdominal tenderness, weak bowel sounds ( $0-1$ beat $/ \mathrm{min})$ and positive shifting dullness. Laboratory examinations reported negative blood and urine amylase. Furthermore, abdominal X-ray examination reported intestinal dilatation of product gas and fluid (Fig. 2). Accordingly, the patient was diagnosed with acute intestinal obstruction. The patient was then fasted and administered conventional 


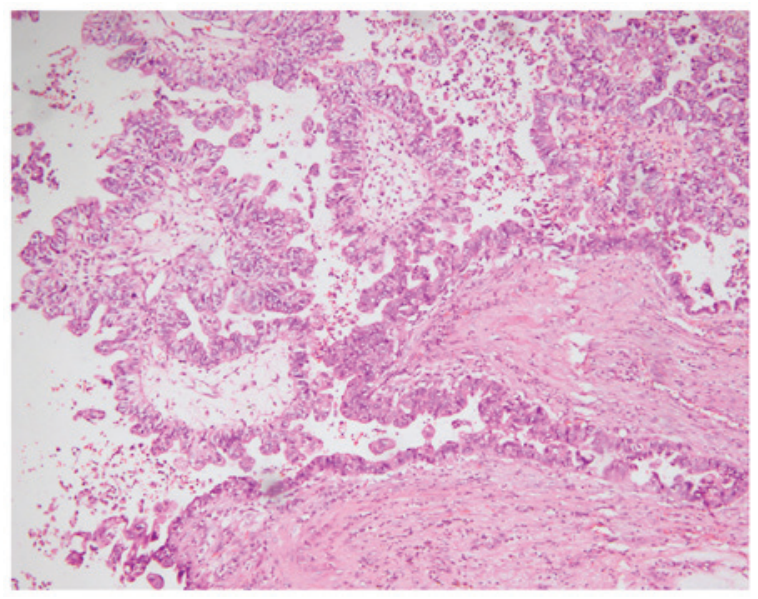

Figure 1. Pathology imaging following hematoxylin and eosin staining demonstrated large and hyperchromatic nuclei of tumor cells, cell growth into the tube and myometrial invasion (magnification, $\mathrm{x} 100$ ).

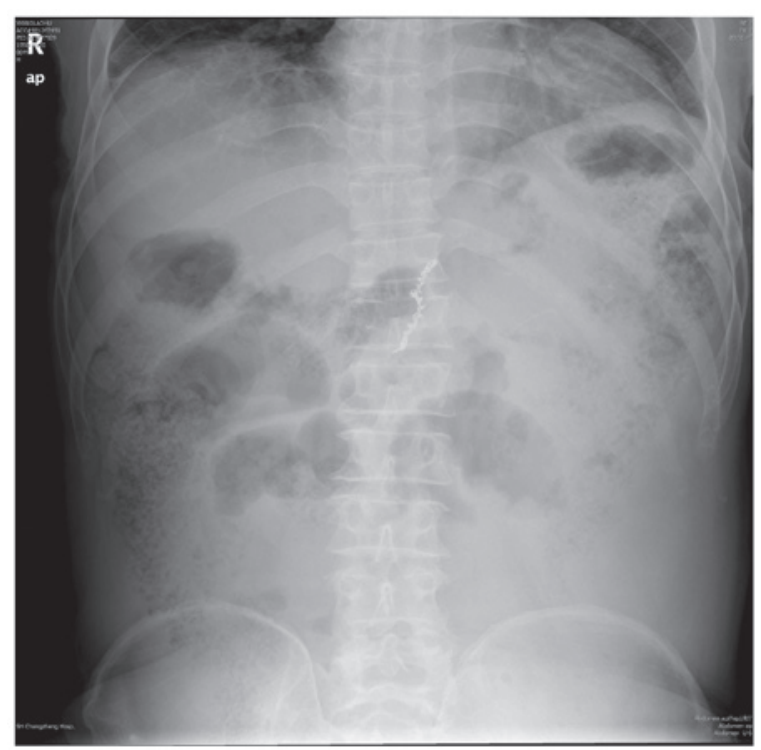

Figure 2. Upright abdominal X-ray revealed intestinal pneumatosis, cavity expansion, the shadow of a small amount of gas in the colon and a large quantity of dung accumulated in the intestinal tract.

treatments, including gastrointestinal decompression, gastrointestinal secretion inhibition, fluid replacement, nutritional support and enema.

The abdominal CT revealed no indications of other acute abdominal diseases, including visceral perforation, rupture or purulent infection, no significant expansion of tumor size or location, or intestinal tumor metastasis. Serum tumor markers declined and a serum potassium was normal. Therefore, the present study hypothesized that the patient may be a rare case of nab-paclitaxel-associated paralytic ileus rather than mechanical resistance, blood flow obstruction or hypokalemia-associated paralytic ileus. Methycobal was futher added to antagonize potential nervous system toxicity caused by nab-paclitaxel. Following active enema treatment, the patient passed a little yellow watery stool on November 29th and defecated with abundant amounts daily from then on. Bloating and abdominal pain were relieved overtly. Flatus also

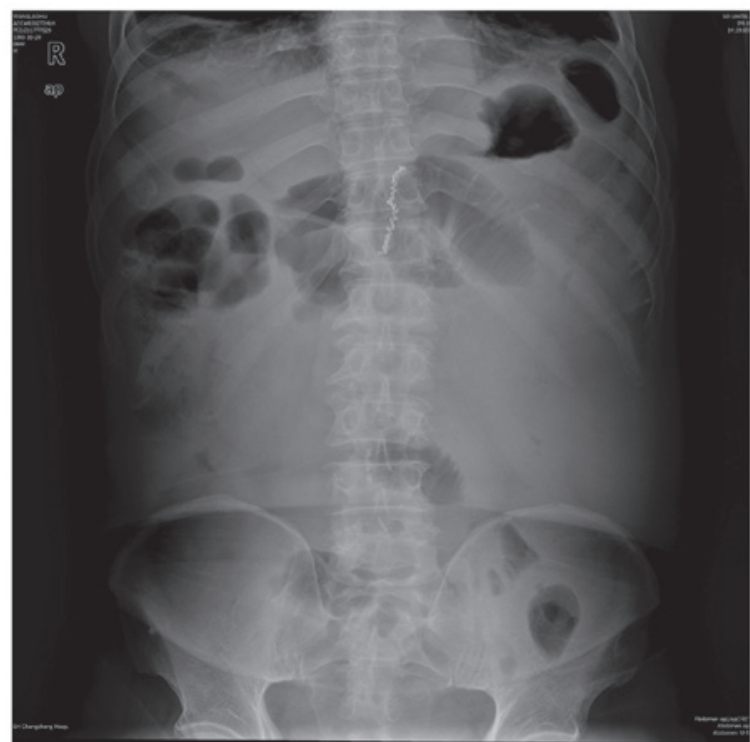

Figure 3. Upright abdominal X-ray demonstrated intestinal pneumatosis, cavity expansion and the shadow of a small quantity of gas in the colon; The intestinal intraluminal stool shadow disappeared.

recovered and bowel sounds returned to 3 times $/ \mathrm{min}$. Another abdominal X-ray examination revealed that the intestinal intraluminal stool shadow disappeared and only a shadow of a small quantity of gas existed in the colon (Fig. 3). Clinical outcomes further supported the diagnosis of paralytic ileus and the patient was discharged 3 days later. Unfortunately, this patient succumbed to mortality, unrelated to the cancer, a week following discharge without any anticancer therapy. Until mortality, his stool remained normal and no more bowl obstruction occurred.

\section{Discussion}

Nab-paclitaxel is an albumin-bound paclitaxel nanoparticle, and it is highly soluble and can easily reach potential tumor tissues through the bloodstream to serve its broad-spectrum antitumor activity. The drug contains no toxic solvents, including polyoxyethylene castor oil or ethanol, which may shorten the elapse of intravenous infusion and reduce the incidence of allergic reactions (2).

Currently, the major reported adverse effects of nab-paclitaxel include cardiac toxicity, nervous system toxicity, muscle and joint pain, gastrointestinal reactions and hematological toxicity (1), whereas bowel obstruction is extremely rare. The present study presented for the first time, to the best of our knowledge, a case of paralytic ileus associated with nab-paclitaxel, which was eliminated following active treatments.

The underlying mechanisms for intestinal obstruction caused by nab-paclitaxel remain to be elucidated. According to previous results from several clinical trials, the incidence of neuropathy of nab-paclitaxel containing regimen ranged between 2.9 and $17 \%(4,15)$, however, all of the neuropathy occurred in peripheral nerve, with no report of autonomic nerve involvement. The present study hypothesized that autonomic nervous system toxicity of nab-paclitaxel may be a probable contributing factor in this case. Additionally, the 
addition of methycobal to conventional treatment $(16,17)$, which possesses trophic action of nerve, further ameliorated the symptoms. The preventive usage of methycobal may be a practical method to reduce the incidence of neuropathy caused by nab-paclitaxel, however, this hypothesis requires further confirmation in clinical practice and clinical trials.

In conclusion, nab-paclitaxel is a novel chemotherapy drug, for which the adverse effects remain to be fully understood. The present study reported for the first time, to the best of our knowledge, that nab-paclitaxel may lead to acute intestinal obstruction in certain cases, and that the obstruction may be induced by nab-paclitaxel-associated autonomic nervous system toxicity. Enough attention to the autonomic nervous toxicity, beside peripheral nervous toxicity, is required in patients using this novel drug.

\section{Acknowledgements}

The present study received the support of the Young Start-up Foundation of Changzheng Hospital (no. 2015CZQN07), the Foundation of Shanghai Municipal Commission of health and family planning (no. 201540174) and the Shanghai Natural Science Foundation (no. 15ZR1414300).

\section{References}

1. Cecco S, Aliberti M, Baldo P, Giacomin E and Leone R: Safety and efficacy evaluation of albumin-bound paclitaxel. Expert Opin Drug Saf 13: 511-520, 2014.

2. Yardley DA: Nab-Paclitaxel mechanisms of action and delivery. J Control Release 170: 365-372, 2013.

3. Yardley DA,Hart L,Bosserman L, Salleh MN, Waterhouse DM, Hagan MK, Richards P, DeSilvio ML, Mahoney JM and Nagarwala Y: Phase II study evaluating lapatinib in combination with nab-paclitaxel in HER2-overexpressing metastatic breast cancer patients who have received no more than one prior chemotherapeutic regimen. Breast Cancer Res Treat 137: 457-464, 2013.

4. Von Hoff DD, Ervin T, Arena FP, Chiorean EG, Infante J, Moore M, Seay T, Tjulandin SA, Ma WW, Saleh MN, et al: Increased survival in pancreatic cancer with nab-paclitaxel plus gemcitabine. N Engl J Med 369: 1691-1703, 2013.
5. Socinski MA, Bondarenko I, Karaseva NA, Makhson AM, Vynnychenko I, Okamoto I, Hon JK, Hirsh V, Bhar P, Zhang H, et al: Weekly nab-paclitaxel in combination with carboplatin versus solvent-based paclitaxel plus carboplatin as first-line therapy in patients with advanced non-small-cell lung cancer: Final results of a phase III trial. J Clin Oncol 30: 2055-2062, 2012.

6. Koizumi W, Morita S and Sakata Y: A randomized Phase III trial of weekly or 3-weekly doses of nab-paclitaxel versus weekly doses of Cremophor-based paclitaxel in patients with previously treated advanced gastric cancer (ABSOLUTE Trial). Jpn J Clin Oncol 45: 303-306, 2015.

7. Guarneri V, Dieci MV and Conte P: Enhancing intracellular taxane delivery: Current role and perspectives of nanoparticle albumin-bound paclitaxel in the treatment of advanced breast cancer. Expert Opin Pharmacother 13: 395-406, 2012.

8. Vishnu P and Roy V: Safety and efficacy of nab-paclitaxel in the treatment of patients with breast cancer. Breast Cancer (Auckl) 5: 53-65, 2011.

9. Matsuoka N, Hasebe H, Mayama T and Fukuchi T: Sub-tenon injections of triamcinolone acetonide had limited effect on cystoid macular edema secondary to nanoparticle albumin-bound-paclitaxel (Abraxane). Case Rep Ophthalmol Med 2015: 181269, 2015.

10. Rivera E and Cianfrocca M: Overview of neuropathy associated with taxanes for the treatment of metastatic breast cancer. Cancer Chemother Pharmacol 75: 659-670, 2015.

11. John P, Butler H and Saif MW: Congestive heart failure secondary to gemcitabine nab-paclitaxel in patients with pancreatic cancer. Anticancer Res 34: 7267-7270, 2014

12. Rahman HT, Yeh S and Bergstrom CS: Cystoid macular edema without leakage secondary to nab-Paclitaxel (Abraxane): Clinical experience with intravitreal bevacizumab. J Ocul Pharmacol Ther 29: 360-362, 2013

13. Casadei Gardini A, Aquilina M, Oboldi D, Lucchesi A, Carloni S, Tenti E, Burgio MA, Amadori D and Frassineti GL: Separate episodes of capillary leak syndrome and pulmonary hypertension after adjuvant gemcitabine and three years later after nab-paclitaxel for metastatic disease. BMC Cancer 13: 542, 2013.

14. Aapro MS, Martin C and Hatty S: Gemcitabine-a safety review. Anticancer Drugs 9: 191-201, 1998.

15. Alberts DS, Blessing JA, Landrum LM, Warshal DP, Martin LP, Rose SL, Bonebrake AJ and Ramondetta LM: Phase II trial of nab-paclitaxel in the treatment of recurrent or persistent advanced cervix cancer: A gynecologic oncology group study. Gynecol Oncol 127: 451-455, 2012.

16. Yagihashi S, Tokui A, Kashiwamura H, Takagi S and Imamura K: In vivo effect of methylcobalamin on the peripheral nerve structure in streptozotocin diabetic rats. Horm Metab Res 14: 10-13, 1982.

17. Ide H, Fujiya S, Asanuma Y, Tsuji M, Sakai H and Agishi Y: Clinical usefulness of intrathecal injection of methylcobalamin in patients with diabetic neuropathy. Clin Ther 9: 183-192, 1987. 